ARTIGO ORIGINAL

ISSN 1677-5090

(C) 2018 Revista de Ciências Médicas e Biológicas

DOI: http://dx.doi.org/10.9771/cmbio.v17i3.28669

\title{
Avaliação in vitro do potencial cariogênico e erosivo de corticosteroide de uso tópico
}

\author{
In vitro evaluation of cariogenic and erosive potential of topic corticosteroid
}

\author{
Tila Fortuna Costa Freire ${ }^{1}$, Elisângela de Jesus Campos², Gabriela Botelho Martins ${ }^{3 *}$ \\ ${ }^{1}$ Doutoranda do Programa de Pós-graduação em Processos Interativos dos Órgãos e Sistemas. Instituto de Ciências \\ da Saúde da Universidade Federal da Bahia (ICS, UFBA).; '2Doutora em Medicina e Saúde.Professora Adjunta do \\ Instituto de Ciências da Saúde da Universidade Federal da Bahia (ICS, UFBA).; ${ }^{3}$ Doutora em Estomatologia Clínica. \\ Professora Adjunta do Instituto de Ciências da Saúde da Universidade Federal da Bahia (ICS, UFBA).
}

\begin{abstract}
Resumo
Introdução: o tratamento com bochechos de dexametasona elixir é bastante descrito na literatura para casos de lesões erosivas e ulceradas em mucosa bucal. Excipientes acrescentados aos medicamentos os tornam mais palatáveis e estáveis, embora possam resultar em efeitos adversos que comprometem a saúde bucal. Objetivo: este estudo propôs-se a avaliar, in vitro, o pH, a acidez total titulável (ATT) e o teor de sólidos solúveis totais (SST) de diferentes marcas de dexametasona elixir disponíveis no mercado da cidade de Salvador, correlacionando-os ao potencial erosivo e cariogênico do medicamento para os dentes. Metodologia: seis marcas (A, B, C, D, E e F) de laboratórios distintos foram incluídas neste estudo. O valor do pH foi aferido utilizando-se pHmetro e agitador magnético; a ATT foi determinada adicionando-se hidróxido de sódio ( $\mathrm{NaOH}$ ) 0,1 N e a aferição do SST foi através de refratômetro. Os dados foram expressos em valores médios e desvios padrão. Resultados: o pH de todas as marcas investigadas apresentou medidas abaixo de 5,5, logo, todas apresentaram potencial erosivo. $\mathrm{Na}$ avaliação da ATT, maior volume de $\mathrm{NaOH} 0,1 \mathrm{~N}$ foi necessário pela marca D para alcançar pH 5,5 e 7,0. Dentre as marcas investigadas, a marca B foi a que apresentou maior teor de SST em sua composição. Conclusão: soluções para uso local de dexametasona elixir possuem potencial erosivo e alto teor de SST, tornando-se, então, importante a orientação de instrução de higiene oral dos pacientes que possuem maior risco de desenvolver alterações dentárias.
\end{abstract}

Palavras-chave: Dexametasona. Erosão Dentária. Cárie Dentária.

\begin{abstract}
Introduction: treatment with mouthwash dexamethasone elixir is sufficiently described in the literature for cases of erosive and ulcerated lesions in oral mucosa. Excipients added to drugs make them more palatable and stable, however, may result in adverse effects that compromise the health of the oral cavity. Aim: this study sets out to evaluate in vitro $\mathrm{pH}$, titratable total acidity (TTA) and the total soluble solids content (TSS) of different brands of dexamethasone elixir available on the market of Salvador city, correlating them to the potential of cariogenic and erosive medicine for the dental units. Methodology: six distinct laboratories brands $(A, B, C$, $D, E$ and $F$ ) were included in this study. The $\mathrm{pH}$ value was assessed using a $\mathrm{pH}$ meter and magnetic stirrer, the TTA was determined by adding sodium hydroxide ( $\mathrm{NaOH}) 0.1 \mathrm{~N}$ and measurement of TSS was made with refractometer. The data were expressed as average and standard deviations. Results: the $\mathrm{pH}$ of all brands investigated presented measures below 5.5, so, all presented erosive potential. In TTA, greater volume of $\mathrm{NaOH} 0,1 \mathrm{~N}$ was required by $\mathrm{D}$ brand to achieve $\mathrm{pH} 5.5$ and 7.0. Among the brands investigated, brand $B$ was the one that presented the highest content of TSS in its composition. Conclusion: solutions for local use of dexamethasone elixir have erosive potential and high content of TSS, becoming so important the guidance of oral hygiene instruction of patients who have higher risk of developing dental changes.

Keywords: Dexamethasone. Dental Erosion. Dental caries.
\end{abstract}

\section{INTRODUÇÃO}

Corticosteroides são drogas amplamente utilizadas, em função de seus efeitos imunossupressivos e anti-inflamatórios, para o tratamento de diversas doenças crônicas e daquelas decorrentes de reações imunes e indesejáveis.

A ulceração aftosa recorrente é uma das condições mais comuns da mucosa oral. Apresenta-se como úlcera única ou múltiplas úlceras dolorosas, que surgem

Correspondência/Corresponding: *Gabriela Botelho Martins - End: Av. Reitor Miguel Calmon S/N sala 413 Canela salvador-Ba CEP: 40231-300 Tel: (71) 3283-8891 - E-mail: gbmartinsba@gmail.com espontaneamente ou após mínimo trauma e cicatrizam espontaneamente (PILZ; CARRARD, 2015). É considerada uma doença inflamatória, com participação do sistema imune. Entre outras lesões imunologicamente mediadas, destacam-se o pênfigo vulgar, o penfigoide benigno das membranas mucosas e o líquen plano. Essas alterações se apresentam como lesões vesículo-bolhosas e representam um grupo heterogêneo de dermatoses com manifestações clínicas bastante variáveis. Tais condições possuem a cavidade oral primordialmente como primeiro sítio acometido, o que exerce importante impacto na qualidade de vida dos pacientes, acarretando quadros álgicos graves e dificuldade para alimentação e fonação, 
a depender da extensão das ulcerações (GONÇALO, 2016). Complicações semelhantes ocorrem em pacientes com mucosite oral decorrente do tratamento quimioterápico e (ou) radioterápico (LESSA, 2012).

O tratamento tópico com bochechos de dexametasona elixir é o mais descrito na literatura em casos de lesões localizadas e brandas na mucosa bucal. Nos casos em que há presença de lesões múltiplas, esse fármaco também é preconizado, devido à sua ação tópica e sistêmica, uma vez que pode ser ingerido após o bochecho (PILZ; CARRARD, 2015).

Devido à incapacidade de algumas crianças e idosos deglutirem formas farmacêuticas sólidas, é comum solicitar ao farmacêutico a preparação de uma forma líquida oral. Os xaropes são preparações aquosas concentradas em açúcar, que servem de veículos de sabor agradável para as substâncias ativas. Os elixires, por sua vez, são soluções hidroalcoólicas que também servem de veículos farmacêuticos (ROWE; SHESKEY; QUINN, 2009).

$\mathrm{O}$ pH das medicações líquidas frequentemente está relacionado com a estabilidade das substâncias ativas, pois pode ocorrer decomposição química em função do $\mathrm{pH}$ inadequado, o que compromete, assim, a ação farmacológica (FERREIRA; SOUZA, 2005).

Os medicamentos pediátricos, em sua maioria, são coloridos, aromatizados e adoçados por meio de um conjunto complexo de adjuvantes, os excipientes, cuja finalidade é a de "mascarar" seu sabor e torná-los mais agradáveis para o consumo da criança. No entanto, a presença de tais componentes podem resultar em efeitos adversos (MARQUEZAN et al., 2007). Várias preparações líquidas ou mastigáveis tornam-se agradáveis ao sabor devido à adição de sacarose, glicose ou frutose como edulcorantes. Esses carboidratos de fermentação rápida, contidos em preparações líquidas, podem interferir no potencial cariogênico dos fármacos em indivíduos com higiene oral deficiente (PAIVA et al., 1991).

Este estudo se propôs a avaliar, in vitro, o pH endógeno, a acidez total titulável (ATT) e o teor de sólidos solúveis totais (SST) de diferentes marcas de um corticosteroide tópico, na forma de elixir, disponíveis no mercado, correlacionando-as com o potencial erosivo e cariogênico dos mesmos.

\section{MATERIAIS E MÉTODOS}

Foi realizado um estudo in vitro, no Laboratório de Bioquímica Oral (LBO) do Instituto de Ciências da Saúde da Universidade Federal da Bahia (ICS/UFBA), sobre o potencial erosivo e cariogênico de corticosteroide de uso tópico de dexametasona na forma de elixir, por meio da determinação das suas propriedades físico-químicas, determinadas através do $\mathrm{pH}$, ATT e SST ( $\left.{ }^{\circ} \mathrm{Brix}\right)$.

Foram encontradas seis marcas de diferentes laboratórios farmacêuticos que comercializam a dexametasona elixir no mercado da cidade de Salvador, a saber: Aché, EMS, Geolab, Medley, NeoQuímica, e TEUTO. Para cada marca comercial, foi atribuída uma codificação conforme listado no Quadro1. A análise bioquímica foi realizada em triplicata, pois, para cada marca comercial, três lotes diferentes foram adquiridos, totalizando 18 amostras estudadas. As análises laboratoriais foram realizadas por um único examinador previamente calibrado e foram anotados em ficha específica.

Quadro 1 - Relação dos constituintes da dexametasona elixir encontradas incluídas neste estudo.

\begin{tabular}{|c|c|c|c|}
\hline Marca & Laboratório & Composição & Lotes \\
\hline \multirow[t]{3}{*}{ Marca A } & Aché & \multirow{3}{*}{$\begin{array}{l}\text { Dexametasona: } 0,1 \mathrm{mg} \\
\text { Glicerol, ácido benzoico, sacarina } \\
\text { sódica di-hidratada, álcool etílico, } \\
\text { água purificada, aroma de cereja } \\
\text { e aroma de menta. }\end{array}$} & L1706854 \\
\hline & & & L1710531 \\
\hline & & & L1705528 \\
\hline \multirow[t]{3}{*}{ Marca B } & EMS & \multirow{3}{*}{$\begin{array}{l}\text { Dexametasona: } 0,1 \mathrm{mg} \\
\text { Sacarina sódica, glicerol, essência } \\
\text { de hortelã, essência de cereja, } \\
\text { vermelho ponceau, álcool etílico, } \\
\text { ácido benzoico, álcool etílico e água } \\
\text { purificada. }\end{array}$} & LOD1023 \\
\hline & & & LOH7449 \\
\hline & & & LOF0931 \\
\hline \multirow[t]{3}{*}{ Marca C } & Geolab & \multirow{3}{*}{$\begin{array}{l}\text { Dexametasona: } 0,5 \mathrm{mg} \\
\text { Glicerol, ácido benzoico, sacarina } \\
\text { sódica, álcool etílico, corante } \\
\text { vermelho ponceau, ciclamato de } \\
\text { sódio, essência de cereja líquida, } \\
\text { essência de menta líquida e água. }\end{array}$} & L1609131 \\
\hline & & & L1605728 \\
\hline & & & L1710007 \\
\hline \multirow[t]{3}{*}{ Marca D } & Medley & \multirow{3}{*}{$\begin{array}{l}\text { Dexametasona: } 0,5 \mathrm{mg} \\
\text { Ácido cítrico, álcool etílico, aroma } \\
\text { de cereja, aroma de menta e } \\
\text { hortelã, benzoato de sódio, } \\
\text { cloreto de sódio, vermelho } \\
\text { ponceau, glicerol, sacarina sódica } \\
\text { di-hidratada, sorbitol, água } \\
\text { deionizada. }\end{array}$} & L17010458 \\
\hline & & & L17090407 \\
\hline & & & L17030425 \\
\hline \multirow[t]{3}{*}{ Marca E } & NeoQuímica & \multirow{3}{*}{$\begin{array}{l}\text { Dexametasona: } 0,1 \mathrm{mg} \\
\text { Sacarina sódica, ciclamato de sódio, } \\
\text { metilparabeno, propilparabeno, } \\
\text { corante vermelho de ponceau, } \\
\text { vanilina, sorbitol, essência de } \\
\text { cereja líquida, álcool etílico e água. }\end{array}$} & LB16F1879 \\
\hline & & & LB16H2731 \\
\hline & & & LB16K2341 \\
\hline \multirow[t]{3}{*}{ Marca F } & TEUTO & \multirow{3}{*}{$\begin{array}{l}\text { Dexametasona: } 0,1 \mathrm{mg} \\
\text { Álcool etílico, sacarina sódica, } \\
\text { ácido benzoico, glicerol, corante } \\
\text { vermelho ponceau, aroma de } \\
\text { cereja e água de osmose reversa. }\end{array}$} & L2766095 \\
\hline & & & L2766100 \\
\hline & & & L2766093 \\
\hline
\end{tabular}

Fonte: o autor

\section{Determinação do pH}

A determinação do $\mathrm{pH}$ dos fármacos foi realizada com temperatura ambiente de $25^{\circ} \mathrm{C}$, utilizando-se pHmetro (Quimis ${ }^{\circledR}$, São Paulo, Diadema, Brasil) combinado com o agitador magnético EV2.017 (EVLAB, Curitiba, Paraná, Brasil). O pHmetro utilizado foi previamente calibrado com as soluções tampão de pH 4,0 e pH 7,0.

\section{Determinação da acidez total titulável (ATT)}

A ATT dos medicamentos foi determinada adicionando-se a essas soluções, gradativamente, volumes de hidróxido de sódio ( $\mathrm{NaOH}$ ) 0,1 N, com a utilização de uma bureta de $50 \mathrm{~mL}$ e sob agitação constante. Os volumes de solução de $\mathrm{NaOH}$ necessários para se atingirem os pHs 5,5 e 7,0 foram registrados, o que correspondeu ao valor da 
ATT de cada marca de medicamento para atingir os níveis de $\mathrm{pH}$ crítico para dissolução do esmalte dentário e pH neutro, respectivamente.

\section{Determinação dos sólidos solúveis totais (SST)}

A determinação dos SST, para a avaliação da concentração em carboidratos, foi realizada por refratometria, método físico, simples e rápido, utilizando-se refratômetro modelo HI 96801 (Hanna ${ }^{\circledR}$, Woonsocket, Rhodelsland, EUA), calibrado com água destilada a $25^{\circ} \mathrm{C}$. As leituras das amostras foram realizadas na escala ${ }^{\circ} \mathrm{Brix}$.

\section{Análise estatística}

Os dados foram tabulados em Excel 2016, e expressos em valores médios de desvios padrão e de coeficiente de variação, os quais foram submetidos a análises descritivas e analíticas.

\section{RESULTADOS}

A composição descrita na bula de cada fármaco, assim como os respectivos lotes analisados, estão apresentados no Quadro 1.

Na tabela 1, encontram-se os valores das médias de $\mathrm{pH}$, ATT e SST, obtidos a partir das triplicatas dos respectivos lotes dos fármacos apresentados no quadro acima citado.

Tabela 1 - Valores de pH, ATT e SST das diferentes marcas comerciais de dexametasona elixir analisadas

\begin{tabular}{|c|c|c|c|c|c|c|c|c|c|c|c|c|}
\hline \multirow[b]{2}{*}{ Laboratório } & \multirow[b]{2}{*}{$\mathrm{pH}$} & \multirow[b]{2}{*}{ DP } & \multirow{2}{*}{$\begin{array}{l}\text { CV } \\
\text { (\%) }\end{array}$} & \multicolumn{6}{|c|}{ ATT $(\mathrm{mL})$} & \multirow{2}{*}{$\begin{array}{c}\text { SST } \\
\text { (Brix) }\end{array}$} & \multirow[b]{2}{*}{ DP } & \multirow{2}{*}{$\begin{array}{l}\text { CV } \\
\text { (\%) }\end{array}$} \\
\hline & & & & $\mathrm{pH} \mathrm{5,5}$ & DP & $\begin{array}{l}\text { CV } \\
\text { (\%) }\end{array}$ & $\mathrm{pH} \mathrm{7,0}$ & DP & $\begin{array}{l}\text { CV } \\
\text { (\%) }\end{array}$ & & & \\
\hline Marca A & 3,37 & 0,01 & 0,2 & 9,1 & 0,32 & 3,5 & 9,9 & 0,76 & 9,9 & 20,9 & 0,00 & 0,0 \\
\hline Marca B & 3,36 & 0,04 & 1,1 & 8,5 & 0,10 & 1,1 & 9,1 & 0,20 & 2,1 & 24,6 & 0,00 & 0,0 \\
\hline Marca C & 3,44 & 0,05 & 1,4 & 13,4 & 0,32 & 2,3 & 14,5 & 0,55 & 3,7 & 21,2 & 0,17 & 0,8 \\
\hline Marca D & 3,29 & 0,03 & 0,9 & 27,3 & 1,90 & 6,9 & 35,0 & 1,72 & 4,9 & 22,7 & 0,05 & 0,2 \\
\hline Marca E & 4,39 & 0,12 & 2,7 & 0,2 & 0,05 & 25,0 & 3,4 & 0,15 & 4,4 & 12,4 & 0,10 & 0,8 \\
\hline Marca F & 3,33 & 0,05 & 1,5 & 9,1 & 0,26 & 2,8 & 9,8 & 0,30 & 3,0 & 13,8 & 0,25 & 1,8 \\
\hline
\end{tabular}

Fonte: o autor

DP - Desvio-padrão

CV - Coeficiente de variação

$\mathrm{O} \mathrm{pH}$ encontrado nas marcas de dexametasona investigadas apresentaram valores próximos entre si e abaixo de 5,5. Sendo assim, todas as amostras apresentaram potencial erosivo. $\mathrm{O}$ menor $\mathrm{pH}$ encontrado foi o da marca comercial $D(3,29)$ e o maior, o da marca $E(4,39)$. Os resultados da ATT - nos pHs de 5,5 e 7,0 - também envolveram essas duas marcas, com os maiores e menores volumes de base, respectivamente. Com relação ao teor de SST presente nos fármacos, observou-se que as medicações da marca $E$ apresentaram menor valor de Brix, em comparação com os demais, enquanto a marca $B$ foi a que apresentou maior valor de oBrix.

Analisando-se os resultados apresentados, pode-se inferir que o fármaco que apresentou maior potencial erosivo, devido ao baixo $\mathrm{pH}$ e ao maior volume de base necessária para reduzir a acidez da medicação, foi a formulação de dexametasona do laboratório da marca $D$.

Com relação à uniformização dos resultados entre os lotes avaliados para cada marca investigada, observou-se que a formulação da marca $E$ foi a que obteve maior variação de $\mathrm{pH}$ entre eles, quando foi avaliado o percentual do coeficiente de variação de suas amostras, em comparação com os demais medicamentos. $O$ coeficiente de variação desta mesma marca, ao se avaliar a ATT, em um pH de 5,5, mostrou-se acima de $15 \%$ e abaixo de $30 \%$, o que indica que os dados coletados possuem uma dispersão média, tornando o resultado regular em torno da média.

\section{DISCUSSÃO}

Excipientes, também conhecidos como adjuvantes farmacêuticos, são substâncias que estão presentes na maioria dos medicamentos. Por serem auxiliares, apresentam propriedades diferentes dos fármacos e podem ser classificados quanto à sua finalidade em: conservantes, corantes, aromatizantes, edulcorantes, espessantes, emulsificantes, estabilizantes, antioxidantes, diluentes, umectantes, solventes, promotores de absorção e matrizes de liberação prolongada (ROWE; SHESKEY; QUINN, 2009).

Tais excipientes deixaram de ser vistos apenas como adjuvantes farmacotécnicos e passaram a constituir componentes indispensáveis para o desenvolvimento de formas farmacêuticas cada vez mais seguras e eficazes, com capacidade de promover a melhoria na qualidade de vida do paciente. Mais de mil produtos são utilizados como excipientes, embora não mais de duzentos estejam descritos na farmacopéia brasileira (PESSANHA et al., 2012). É possível inferir que a diferença nos valores dos parâmetros avaliados dos fármacos investigados no presente estudo pode ter relação direta com os constituintes encontrados para formulação das dexametasonas sob a forma de elixir, nos diferentes laboratórios.

Os excipientes são incorporados aos constituintes dos fármacos com a finalidade de tornar as medicações mais palatáveis e toleráveis aos pacientes. Um estudo se 
propôs avaliar os níveis de tolerância e palatabilidade da dexametasona elixir e da prednisolona em comprimidos, triturados e dispersos em líquido, e sob a forma de xarope, com 122 crianças da Arábia Saudita e 133 crianças do Reino Unido. A palatabilidade foi avaliada utilizando-se a escala facial Hedonic de 5 pontos e tolerabilidade por questionamento direto aos pacientes e pais. Observou-se que, em ambos os países, a dexametasona elixir foi considerada mais palatável em comparação com a prednisolona e que os níveis de palatabilidade melhoraram com o passar dos dias e o uso de doses subsequentes dos dois fármacos. Ainda segundo o estudo, em ambos os países, a dexametasona elixir também foi mais tolerada pelas crianças por ter apresentado menor número de efeitos colaterais descritos, como vômitos, náuseas e dor abdominal (ALJEBAB et al., 2018).

Agentes acidulantes desempenham várias funções $e$, dentre as principais, a de aromatizantes, reguladores de $\mathrm{pH}$, agentes tamponantes e agentes de fermentação. Baixos índices de $\mathrm{pH}$ nos fármacos podem estar relacionados a tais agentes, presentes nas formulações, como o ácido cítrico, mais utilizado na indústria farmacêutica. Esse ácido é considerado um potente agente erosivo, devido à capacidade de quelar o cálcio da hidroxiapatita e, consequentemente, aumentar a velocidade de dissolução do esmalte dentário frente a desafios com ácido. Tal ação quelante permanece ativa, mesmo que ocorra a elevação do $\mathrm{pH}$ na superfície dentária (LUSSI; JAEGGI, 2006; NEVES; PIERRO; MAIA, 2007). Esse componente, presente na formulação da marca $\mathrm{D}$, possivelmente é o responsável pelo menor $\mathrm{pH}$ encontrado no medicamento, que utiliza este ácido como um de seus constituintes, o qual estava ausente em todas as demais formulações investigadas no presente estudo.

Em quatro das formulações analisadas, observou-se a presença de ácido benzoico utilizado como conservante. Ressalte-se que a marca $\mathrm{E}$, que apresentou os menores valores de $\mathrm{pH}$ inicial e a menor resistência no ATT para chegar aos pHs 5,5 e 7,0 respectivamente, não apresentou nenhuma das duas substâncias, o que parece ter interferido nesse resultado.

Estudos in vitro (MOMESSO et al., 2009; MOREIRA et al., 2012) mostram que o esmalte dentário, exposto a soluções com pH abaixo de cinco, apresenta sua superfície alterada, formando lesões macro e microscopicamente semelhantes à erosão que se desenvolve na cavidade bucal. É consenso, na literatura (BARATIERI et al., 2002; MOMESSO et al., 2009), que o $\mathrm{pH}$ crítico para o esmalte dentário é de aproximadamente 5,5. Logo, pode-se afirmar que todos os corticosteroides tópicos investigados no presente estudo apresentaram potencial erosivo. Todavia não se deve desconsiderar que a erosão dentária também é dependente da dose e frequência de uso do medicamento e hábitos de higiene oral do indivíduo.

A saliva participa da formação do biofilme na superfície do esmalte dentário, uma ação protetora que reduz o efeito desmineralizante das medicações ácidas (NEKRASHEVYCH; STOSSER, 2003). No entanto, apesar de as substâncias com baixa ATT serem potencialmente neutralizadas por fluidos orais, aquelas com alta ATT causam queda prolongada do $\mathrm{pH}$ e, consequentemente, maior desmineralização dos tecidos dentais (COSTA et al., 2004). A ATT é uma medida do peso percentual de ácido presente em uma solução, calculada a partir do volume de hidróxido de sódio $(\mathrm{NaOH})$ ou hidróxido de potássio (KOH) necessário para neutralizar as espécies ácidas (CAVALCANTI et al., 2013).

Neste estudo, o $\mathrm{NaOH}$ 0,1 $\mathrm{N}$ foi utilizado na avaliação do volume necessário para se alcançarem os níveis de $\mathrm{pH}$ 5,5 e 7,0 . As variações de volume de base encontradas nas seis marcas de corticosteroides tópicos investigadas oscilou de 0,2 a $27,3 \mathrm{~mL}$, até alcançar o pH de 5,5 e de 3,4 a $35 \mathrm{~mL}$, para alcançar o $\mathrm{pH}$ de 7,0 .

A propriedade de uma substância em ser capaz de resistir à modificação do seu $\mathrm{pH}$ é chamada de capacidade tampão (FOOD INGREDIENTS BRASIL, 2011). O ácido cítrico é o acidulante que possui a maior capacidade tampão, sendo tal ácido encontrado no elixir de dexametasona da formulação da marca $D$. A presença desse componente nessa marca de fármaco talvez explique a necessidade de maior volume de base necessária para a elevação do $\mathrm{pH}$ durante o teste da ATT.

Em um estudo in vitro, que avaliou 22 medicações pediátricas - antibióticos, anti-histamínicos, anti-helmínticos, anti-inflamatórios não esteroidais e corticosteroides -, os autores constataram que, em todas as classes de medicamentos, havia fármacos considerados potencialmente erosivos para os tecidos dentais. Apenas nove dos 22 fármacos investigados possuíam pH superior a 5,5. De acordo com esses resultados, os autores sugeriram que o controle e o acompanhamento dos pacientes que fazem uso dessas medicações devem ser estabelecidos de acordo com o potencial erosivo de cada medicação em particular, e a administração dos fármacos deve ser controlada e acompanhada de uma higiene bucal adequada (CAVALCANTI et al., 2013).

A determinação da quantidade de SST através da refratometria tem sido um método utilizado na literatura (CAVALCANTI et al., 2013; SOUSA et al., 2010) para a determinação do potencial cariogênico de uma determinada solução. Os SST contidos em um produto representam o total de todos os sólidos dissolvidos na água, começando com açúcar, sais, proteínas e ácidos (MORAES, 2018)

Um estudo in situ investigou a relação da concentração de sacarose e seu potencial cariogênico no esmalte dentário. Nele, 12 participantes voluntários utilizaram aparelhos palatinos intraorais, cada um com 4 blocos de esmalte dentário que foram submetidos a diferentes tratamentos com soluções de sacarose em concentrações que variavam entre 1, 5, 10, 20 e 40\%, por 8 vezes ao dia. Os achados dos autores sugerem que o limiar da concentração da solução de sacarose, para a formação de um biofilme cariogênico, é de $5 \%$, apresentando o mesmo potencial cariogênico observado para 10 e $20 \%$ de solução de sacarose (AIRES et al., 2006). 
Importante é verificar que, nas formulações farmacêuticas avaliadas, nenhuma marca apresentou sacarose em suas bulas. Foi observada a presença de edulcorantes, ou substitutos de açúcar, a saber: sacarina sódica e ciclamato de sódio, além de sorbitol. Assim, não se pode correlacionar o possível potencial cariogênico desses medicamentos à sacarose. Contudo, os valores relevantes de SST encontrados nas análises realizadas podem estar associados aos aromatizantes e essências presentes nas formulações, uma vez que estes podem ser fonte de carboidratos, a depender de sua origem e classificação (ANVISA, 2007). Esses ingredientes são usados para melhorar o sabor dos medicamentos. Seu quantitativo é segredo industrial e, portanto, não vêm especificado nas bulas (BALBANI; STELZER; MONTOVANI, 2006), o que dificulta o estabelecimento de correlação, não somente com a presença, como também a quantidade do agente presente e seu respectivo potencial cariogênico.

A análise dos teores de sólidos solúveis totais, realizada por Sousa et al. (2010), revelou que, entre a clemastina, o dicloridrato de cetirizina, o maleato de dexclorfeniramina e a dexametasona, a dexametasona foi o fármaco com menor grau @Brix $(13,25 \%)$, em comparação com os demais fármacos, que apresentaram oBrix elevado (de $37,45 \%$ a $63,66 \%$ ). No presente estudo, observou-se que houve variação no conteúdo de SST presente nos corticosteroides tópicos, a depender do laboratório que formule e comercialize tal fármaco. Entretanto, considerando-se que o ${ }^{\circ}$ Brix variou de $13,8 \%$ a $24,6 \%$, pôde-se constatar que todos os fármacos apresentaram excipientes que interferiram no quantitativo de sólidos totais presentes. É possível que a presença dos aromatizantes possa justificar esses resultados.

Nos elixires analisados neste estudo, foram observados aromas de cereja, menta e hortelã, além das essências de cereja, menta e hortelã. Em um estudo que avaliou a presença de aromatizantes em 73 formulações terapêuticas, foi possível observar que $83 \%$ das amostras possuíam aromas de frutas e $22 \%$ apresentaram outros aromas ou essências, como anis, canela, cassis, licor, mel, mentol, rum, dentre outros. Os autores também observaram que 16 dos 73 medicamentos analisados possuíam duas essências, e sete deles, três essências (BALBANI; STELZER; MONTOVANI, 2006).

No combate à cárie, um dos principais aliados é a escovação. Além de desestruturar o biofilme formado sobre a estrutura dentária, os dentifrícios possuem flúor, o qual é capaz de interferir na dinâmica do processo de cárie, reduzindo a quantidade de minerais perdidos quando do fenômeno da desmineralização e ativando a quantidade de reposição mineral quando da remineralização salivar. Logo, esse componente se torna eficiente na redução da progressão da doença cárie. Quando o açúcar é convertido em ácidos pelo biofilme dental e atinge $\mathrm{pH}$ crítico para a dissolução dos minerais à base de apatita, a presença de flúor no meio auxilia na formação de fluorhidroxiapatita, minimizando as perdas minerais (BARATIERI et al., 2002).
Neves, Pierro e Maia, em 2007, entrevistaram 86 responsáveis por pacientes pediátricos acerca da higiene oral de seus filhos e do uso de medicações infantes. Segundo os autores, a maioria dos responsáveis não realizava higiene bucal de seus filhos após a ingestão de medicamentos líquidos e apenas 08 deles haviam sido instruídos previamente sobre a necessidade de higienização bucal após ingestão de tais fármacos.

O presente estudo mostrou que fármacos prescritos de forma rotineira para o tratamento tópico de condições como estomatites aftosas recorrentes, mucosites decorrentes do tratamento antineoplásico e lesões vesículo-bolhosas ocasionadas por doenças autoimunes, entre outras, podem provocar efeitos deletérios indesejados na cavidade oral. Observou-se que a correta orientação de higiene oral para os usuários desses fármacos é de fundamental importância para prevenir a perda da estrutura dentária.

\section{CONCLUSÕES}

Este estudo possibilitou identificar o potencial erosivo e o alto teor de SST das formulações de dexametasona elixir disponíveis no mercado brasileiro. Embora tenham sido obtidos valores de ATT e SST relevantes na maioria dos medicamentos estudados, não foi possível estabelecer a possibilidade de potencial cariogênico nas formulações avaliadas, pela falta de informações referentes a todos os constituintes presentes na bula. Isto reforça a necessidade de que os profissionais da saúde forneçam recomendações para pacientes com alto risco de erosão e cárie dentária, auxiliando na prevenção dessas possíveis complicações.

\section{REFERÊNCIAS}

AIRES, C. P. et al. Effect of sucrose concentration on dental biofilm formed in situ and on enamel demineralization. Caries res., Basel, v. 40, p.28-32, 2006.

ALJEBAB, F. et al. Observational study on the palatability and tolerability of oral prednisolone and oral dexamethasone in children in Saudi Arabia and the UK. Arch. dis. child., London, v.103, p. 83-88, 2018.

BALBANI, A.P.S.; STELZER. L.B.; MONTOVANI, J.C. Excipiente de medicamentos e as informações da bula. Rev. bras. otorrinolaringol., Rio de Janeiro, v.72, n.3, p. 400-406, 2006.

BARATIERI, L. N. et al. Odontologia restauradora: fundamentos e possibilidades. São Paulo: Santos, 2002. p. 31-68.

BRASIL. Ministério da Saúde. Agência Nacional de Vigilância Sanitária (ANVISA). Resolução de diretoria colegiada - RDC n 2, de 15 de jan. de 2007.

CAVALCANTI, A. L. et al. In vitro evaluation of diferente physicochemical properties of pediatric medicines. Pesq. Bras. Odontoped. Clin. Integr., João Pessoa, v.13, p. 69-75, Jan./Mar. 2013.

COSTA, C.C. et al. Análise do pH endógeno, da acidez e da concentração de sacarose de medicamentos pediátricos. Rev. odonto ciênc., Porto Alegre, v.19, n.44, p.164-169, 2004.

FERREIRA, A. O.; SOUZA, G. F. Preparações orais líquidas. São Paulo: Pharmabooks, 2005. 
FOOD INGREDIENTS BRASIL. Dossiê acidulantes. Purac, 2011. n. 19 p. 24-30.

GONÇALO, R. I. C. Doenças autoimunes bolhosas com manifestação em mucosa oral: estudo retrospectivo e de acompanhamento. 2016. 38f. Monografia (Graduação) - Departamento de Odontologia, Universidade Federal do Rio Grande do Norte, Natal, 2016.

LESSA, L. S. A importância do cirurgião dentista antes e após o transplante de medula óssea: uma revisão de literatura. 2012.55f. Monografia (Graduação) - Universidade Federal de Santa Catarina, Florianópolis, 2012.

LUSSI, A.; JAEGGI, T. Chemical factors. In: LUSSI, A. Dental Erosion. Monogr. Oral sci., Basel, v.7, p. 77-87, 2006.

MARQUEZAN, M. et al. Medicamentos utilizados por pacientes odontopediátricos e seu potencial cariogênico. RPG, Rev. Pós-Grad., São Paulo, v.13, n. 4, p.334-339, 2007.

MOMESSO, M.G.C. et al. Estudo das alterações em esmalte de dentes decíduos após exposição a bebidas disponíveis no mercado. Stomatos, Canoas, v.15, n. 29, p. 4-15, jul./dez. 2009.

MORAES, R. R. Refratometria. 2018. Disponível em: http:<//www.fapepi. pi.gov.br/novafapepi/ciencia/documentos/ >. Acesso em: 13 jun. 2018.

MOREIRA, M. S. C. et al. Avaliação microestrutural do esmalte bovino exposto a bebidas lácteas fermentadas e propriedades físico químicas. Pesq. Bras. Odontoped. Clín. Integ., João Pessoa, v.12, n. 2, p. 161 167, abr./jun. 2012.

NEKRASHEVYCH, Y.; STOSSER, L. Protective influence of experimentally formed salivary pellicle on enamel erosion. Caries res., Basel, v.37, p.225-231, 2013.

NEVES, B. G.; PIERRO, V. S. S.; MAIA, L. C. Percepções e atitudes de responsáveis por crianças frente ao uso de medicamentos infantis e sua relação com cárie e erosão dentária. Cienc. Saúde Colet., Rio de Janeiro, v.12, n.5, p.1295-1300, 2007.

PAIVA, S. M. et al. Cariogenicidade dos medicamentos. RGO, Porto Alegre, v. 39, n. 5, p.336-338, 1991.

PESSANHA, A. F. V. et al. Influência dos excipientes multifuncionais no desempenho dos fármacos em formas farmacêuticas. Rev. bras. farm., Rio de Janeiro, v.93, n.2, p. 136-145, 2012.

PILZ, C.; CARRARD, V. C. Ulceração aftosa recorrente. Rio Grande do Sul: Regula SUS, 2015.

ROWE, R. C.; SHESKEY, P.J.; QUINN, M.E. Handbook of pharmaceutical excipients. 6. ed. Londres: Pharmaceutical Press, 2009.

SOUSA, R. I. M. et al. Potencial erosivo e cariogênico de anti-histamínicos de uso infantil. RFO, Passo Fundo, v.15, n. 3, p.255-260, set./dez. 2010.

Submetido em: $19 / 11 / 2018$

Aceito em: 29/11/2018 